\title{
Genetic variant I148M in PNPLA3 is associated with the ultrasonography-determined steatosis degree in a Chinese population
}

\author{
Yiling $\mathrm{Li}^{{ }^{*+}}$, Chao Xing ${ }^{2^{*+}}$, Zhong $\operatorname{Tian}^{3}$ and Hung-Chih $\mathrm{Ku}^{2}$
}

\begin{abstract}
Background: Nonalcoholic fatty liver disease (NAFLD) is an escalating medical problem worldwide. A nonsynonymous single nucleotide polymorphism rs738409 (1148M) in patatin-like phospholipase domain-containing protein 3 (PNPLA3) predisposes susceptibility to NAFLD; however, its association with steatosis grade is inconsistent in the literature. In particular, there was no significant association found between $1148 \mathrm{M}$ and steatosis grade in two East Asian-based studies. In this study we aim to investigate whether 1148M is associated with the ultrasonography-determined steatosis degree in Chinese adults.

Methods: 203 NAFLD cases and 202 matched controls were recruited. Cases were classified into mild, moderate and severe fatty liver by ultrasonography. Association between 1148M and the ultrasonography-determined steatosis degree as well as other clinical parameters was evaluated.

Results: The $1148 \mathrm{M}$ variant was associated with the ultrasonography-determined steatosis degree with the $\mathrm{M}$ allele frequencies being $0.32,0.54$, and 0.87 in mild $(n=105)$, moderate $(n=83)$, and severe $(n=15)$ cases, respectively $\left(P\right.$-value $\left.=7.6 \times 10^{-8}\right)$. We also confirmed the interaction between $1148 \mathrm{M}$ variation and body mass index towards elevated plasma alanine aminotransferase levels in cases $\left(P\right.$-value $\left.=4.4 \times 10^{-4}\right)$.
\end{abstract}

Conclusion: The PNPLA3 I148M variant is associated with the ultrasonography-determined steatosis degree in Chinese population.

Keywords: PNPLA3, NAFLD, Ultrasonography, Steatosis grade

\section{Background}

Nonalcoholic fatty liver disease (NAFLD) is an escalating medical problem worldwide. It affects $20-34 \%$ of the population in Western countries [1]; although the prevalence of NAFLD is somewhat lower in East Asia-25\% in Japan [2], 18\% in South Korea [3], and 15\% in China [4] - its incidence has increased rapidly with a growing prevalence in young generations during the last two decades $[5,6]$.

NAFLD is a multifactorial disorder associated with obesity, insulin resistance, type 2 diabetes, and dyslipidemia

\footnotetext{
*Correspondence: ly172@hotmail.com; chao.xing@utsouthwestern.edu ${ }^{\dagger}$ Equal contributors

'Department of Gastroenterology, First Affiliated Hospital of China Medical University, Shenyang 110001, China

${ }^{2}$ McDermott Center for Human Growth and Development, University of Texas Southwestern Medical Center at Dallas, Dallas, TX 75390, USA Full list of author information is available at the end of the article
}

$[1,7]$. Familial clustering of steatosis, nonalcoholic steatohepatitis, and cryptogenic cirrhosis suggests that genetic factors contribute to the susceptibility to NAFLD $[8,9]$. Multiple genes have been reported to be associated with NAFLD by candidate gene studies (for a review see [10]), and, more recently, by genome-wide association studies [11-13]. A nonsynonymous sequence variation (rs738409) in patatin-like phospholipase domain-containing protein 3 (PNPLA3) that substitutes methionine for isoleucine at residue 148 (I148M) was first reported to be associated with hepatic triglyceride content in a genome-wide screen [11]; subsequently its association with NAFLD was confirmed in multiple ethnic and geographic groups (for a review see [14]) including the East Asian populations [15-19]. The I148M variant was also reported to be associated with the histological severity of NAFLD (for a review see [20]); however, in two East

\section{Biomed Central}

(c) 2012 Li et al.; licensee BioMed Central Ltd. This is an Open Access article distributed under the terms of the Creative Commons Attribution License (http://creativecommons.org/licenses/by/2.0), which permits unrestricted use, distribution, and reproduction in any medium, provided the original work is properly cited. 
Asian-based studies, there was no significant association between I148M and steatosis stage $[15,16]$. In particular, in a sample of 112 Chinese NAFLD patients I148M was found to be associated with fibrosis but not steatosis grade [16]. In this study we investigated the association between I148M and the ultrasonography-determined steatosis degree in a larger collection of Chinese NAFLD patients.

Although the PNPLA3 I148M variant is not associated with body-mass-index (BMI) in the general population $[11,13]$, it was shown that morbid obesity exposed the association between the $148 \mathrm{M}$ allele and plasma alanine aminotransferase (ALT) levels in both obese adults [21] and children [22,23]. Further it was shown in obese children the association between I148M and ALT levels was due to the interaction with abdominal fat [24]. More recently it was shown the $148 \mathrm{M}$ allele interacted with obesity towards type 2 diabetes susceptibility [25]. In this study we also attempted to replicate the interaction between I148M and BMI on ALT levels and to investigate whether it contributes to NAFLD susceptibility.

\section{Methods}

A total of 203 unrelated adults with NAFLD were recruited from an outpatient liver clinic at the First Affiliated Hospital of China Medical University in Shenyang, China, between October 2010 and April 2011. The patients were confirmed to have hepatic steatosis by liver ultrasonography and classified into three categories-mild, moderate, and severe-according to established criteria [26]. In particular, five criteria were used to diagnose NAFLD: 1) diffuse enhancement of near field echo in the hepatic region and gradual attenuation of the far field echo; 2) unclear display of intra-hepatic lacuna structure; 3) mild to moderate hepatomegaly with a round blunt border; 4) reduction of blood flow signal in the liver; and 5) unclear or non-intact display of envelop of right liver lobe and diaphragm. Patients meeting criterion 1 and any one of criteria 2-4 were classified as mild; patients meeting criterion 1 and any two of criteria 2-4 were classified as moderate; and patients meeting criteria 1, 5, and any two of criteria 2-4 were classified as severe [26]. All the examinations were performed by one experienced radiologist, who was unaware of the patients' clinical details and laboratory findings, using a GE Vivid7 ultrasound machine (GE Healthcare, Horten, Norway) equipped with a GE 4C curved array transducer (GE H4904PC). Secondary causes of steatosis-ethanol intake, total parenteral nutrition, hepatitis $\mathrm{B}$ and Hepatitis $\mathrm{C}$ virus, autoimmune liver disease, hemochromatosis, alpha1-antitrypsin deficiency, Wilson's disease, use of drugs that promote steatosiswere ruled out. A total of 202 ethnicity-matched controls with normal liver enzyme levels and no steatosis by ultrasonography were recruited from primary care outpatient clinics at the same institution. Written informed consent was obtained using a protocol approved by the ethics committee of the First Affiliated Hospital of China Medical University.

Age and sex were self-reported. BMI was calculated according to the measured height and weight at the time of recruitment. Venous blood samples were obtained from the subjects after an overnight fast (12 hours). Plasma ALT, aspartate aminotransferase (AST), $\gamma$ glutamyltransferase (GGT), fasting blood glucose (FBG), triglycerides, high density lipoprotein cholesterol (HDL-C) and low density lipoprotein cholesterol (LDL-C) levels were measured using an automated analyzer. Genomic DNA was extracted from leukocytes using AxyPrep Whole Blood Genomic DNA Miniprep Kit (Axygen Biosciences, USA). The I148M variant was genotyped using a TaqMan assay on a 7900HT Fast Real-Time PCR instrument (Applied Biosystems, Foster City, CA) at $50^{\circ} \mathrm{C}$ for $2 \mathrm{~min}, 95^{\circ} \mathrm{C}$ for $10 \mathrm{~min}$, and then 40 cycles of $95^{\circ} \mathrm{C}$ for $15 \mathrm{sec}$ and $60^{\circ} \mathrm{C}$ for $1.5 \mathrm{~min}$. Hardy-Weinberg equilibrium (HWE) was examined by Pearson's $\chi^{2}$ goodness-of-fit test in cases and controls, respectively.

Comparisons of demographic and clinical features between cases and controls were performed by a twosample $t$-test for continuous variables and by a proportion test for categorical variables. Comparisons between caterogies of cases were performed by analysis of variance. Linear regression models were fit to test association between genotype and continuous phenotypes, and logistic regression models were fit to test association between genotype and categorical phenotype. Bonferroni correction was performed to correct for multiple testing. The genotypic value was coded in an additive manner, i.e., 0, 1, and 2 denoted II, IM, and MM genotypes, respectively. The interaction between I148M and BMI for a continuous trait-ALT-was tested by examining the interaction term in a linear regression model; the interaction for an ordinal trait-the ultrasonographydetermined steatosis degree-was tested by examining the interaction term in an ordered logistic regression model; the interaction for a binary trait-NAFLD-was tested either by examining the interaction term in a logistic regression model using both cases and controls, or by examining the association between I148M and BMI using only cases. We estimated the power to detect interaction a priori given the sample size; when there was no significant interaction detected, we also estimated sample sizes required to achieve the power of 0.8 . The power analyses were performed for a quantitative trait [27] and for a binary trait in both case-control $[28,29]$ and case-only [30] designs. All analyses were performed using $\mathrm{R}$ [31] except that power analysis in 
a case-control design was performed using POWER V3.0 $[28,29]$.

\section{Results}

The comparisons of demographic, clinical, and genetic characteristics between the 203 NAFLD cases and 202 controls were reported elsewhere [17]. Here we focused on comparing the characteristics among cases with the different ultrasonography-determined steatosis degrees, as summarized in Table 1. Based on liver ultrasonography the 203 NAFLD cases were classified into three categories-mild degree $(n=105)$, moderate degree $(n=83)$, and severe degree $(n=15)$. The 15 severe cases were on average younger than the mild and moderate cases $(38.9 \pm 13.9$ years versus $47.6 \pm 13.3$ and $47.4 \pm 13.2$ years, respectively). BMI increased with the ultrasonography-determined steatosis degree $(P<0.01)$. Liver enzyme levels ALT and AST increased with the steatosis degree $(P$-values $<0.01)$ whereas GGT levels did not $(P>0.10)$. Triglycerides levels showed a marginal increase with the increase of the steatosis degree (nominal $P$-value $=0.03$ ), whereas LDL-C and HDL-C levels did not $(P$-values $>0.10)$. FBG levels were higher in the 15 severe cases, though not statistically significant $(P$-value $>0.10)$.

The distribution of the I148M variant in cases and controls was summarized in Table 2. In controls it was in HWE ( $P$-value $=0.59)$, which guaranteed the

\begin{tabular}{|c|c|c|c|c|}
\hline \multirow[t]{2}{*}{ Variable } & \multicolumn{3}{|c|}{ Steatosis grade } & \multirow[t]{2}{*}{$P$-value ${ }^{\ddagger}$} \\
\hline & Mild & Moderate & Severe & \\
\hline Sample size & 105 & 83 & 15 & \\
\hline Age (years) & $47.6 \pm 13.3$ & $47.4 \pm 13.2$ & $38.9 \pm 13.9$ & 0.06 \\
\hline BMI $\left(\mathrm{kg} / \mathrm{m}^{2}\right)$ & $26.1 \pm 3.7$ & $27.2 \pm 4.4$ & $30.5 \pm 5.3$ & $<0.01$ \\
\hline ALT (IU/L) & $30.9 \pm 21.3$ & $50.1 \pm 36.1$ & $106.3 \pm 78.5$ & $<0.01$ \\
\hline AST (IU/L) & $28.8 \pm 26.9$ & $29.5 \pm 13.4$ & $59.8 \pm 62.46$ & $<0.01$ \\
\hline GGT (IU/L) & $63.0 \pm 83.8$ & $55.8 \pm 48.6$ & $78.2 \pm 47.7$ & $>0.10$ \\
\hline Triglycerides (mmol/L) & $1.8 \pm 1.3$ & $2.3 \pm 1.5$ & $2.4 \pm 1.8$ & 0.03 \\
\hline LDL-C (mmol/L) & $3.4 \pm 1.0$ & $3.3 \pm 1.1$ & $3.1 \pm 1.2$ & $>0.10$ \\
\hline $\mathrm{HDL}-\mathrm{C}(\mathrm{mmol} / \mathrm{L})$ & $1.3 \pm 0.4$ & $1.4 \pm 0.8$ & $1.1 \pm 0.2$ & $>0.10$ \\
\hline FBG $(\mathrm{mmol} / \mathrm{L})$ & $6.0 \pm 1.3$ & $6.0 \pm 1.1$ & $6.7 \pm 1.9$ & $>0.10$ \\
\hline \multicolumn{5}{|l|}{ PNPLA3-I148M } \\
\hline$\|$ & 52 & 18 & 0 & $7.6 \times 10^{-8}$ \\
\hline IM & 39 & 41 & 4 & \\
\hline MM & 14 & 24 & 11 & \\
\hline
\end{tabular}

${ }^{\dagger}$ Data are described by mean \pm standard deviation for continuous variables. ${ }^{\ddagger}$ For demographic and clinical variables nominal $P$-values were calculated by an F-test using ANOVA; for I148M the $P$-value was calculated by Fisher's exact test. $P$-values for BMI, ALT, and AST remained $<0.01$ after Bonferroni multiple testing correction ( 9 tests). genotyping quality, but in cases it was out of HWE $(P$-value $=0.02)$, which indicated its association with the disease status [32]. We previously showed the I148M variant was associated with the NAFLD affection status with the minor allele $M$ being more frequent in cases than in controls $\left(0.45\right.$ versus $0.31 ; P$-value $\left.=1.5 \times 10^{-4}\right)$ [17]. This variant was also associated with the ultrasonography-determined steatosis degree with the $\mathrm{M}$ allele frequencies being $0.32,0.54$, and 0.87 in mild, moderate, and severe cases, respectively $(P$-value $=$ $\left.7.6 \times 10^{-8}\right)$. We further investigated the association between I148M and other clinical parameters in cases and controls, respectively (Table 2). In cases, as the copy number of $M$ allele increased, ALT levels significantly increased (nominal $P$-value $=1.1 \times 10^{-7}$, Bonferroni corrected $P$-value $\left.=2.0 \times 10^{-6}\right)$, and AST showed a tendency of increase but not statistically significant (nominal $P$-value $=0.07$ ), whereas LDL-C levels showed a tendency of decrease (nominal $P$-value $=4.1 \times 10^{-3}$; Bonferroni corrected $P$-value $=7.4 \times 10^{-2}$ ). None of the clinical features were significantly associated with I148M in controls.

There was no statistically significant association between I148M and BMI in either cases or controls, or the combined sample $(P$-values $>0.10)$. Therefore we investigated their interaction towards ALT levels and NAFLD susceptibility assuming independence between them. Given the allele frequency of I148M variant in cases and the distributions of ALT levels and BMI in each genotypic category, the power to detect a significant interaction between I148M and BMI at the level of 0.05 was greater than 0.99 [27], and we did detect an interactive effect between them towards elevated ALT levels in cases $\left(P\right.$-value $\left.=4.4 \times 10^{-4}\right)$ by a linear regression model. Stratifying the cases into quarters by BMI, it showed the MM genotype interacted with high BMI to elevate ALT levels (Figure 1). The power to detect an intercation between I148M and BMI towards NAFLD susceptibility was estimated to be 0.12 for a case-control design $[28,29]$ and 0.20 for a case-only design [30], respectively, and we did not detect any significant interaction given the data $(P$-values $>0.10)$. To detect a significant interaction at the level of 0.05 with a power of 0.8 , it would need a sample size of either 2,362 cases or 1,316 cases and 1,316 controls. There was no significant interaction between I148M and BMI towards elevated AST levels or the ultrasonography-determined steatosis degree $(P$-values $>0.10)$.

\section{Discussion}

It is well recognized the PNPLA3 I148M variant predisposes susceptibility to NAFLD and it is associated with the histological severity of NAFLD. However, some studies showed it was associated with steatosis grade [33-37], whereas in two East Asian-based studies, there was no 
Table 2 Association between PNPLA3 I148M and clinical parameters in NAFLD cases and controls ${ }^{\dagger}$

\begin{tabular}{|c|c|c|c|c|c|c|c|c|}
\hline \multirow[t]{2}{*}{ Variable } & \multicolumn{3}{|c|}{ Cases } & \multirow[t]{2}{*}{$P$-value ${ }^{\ddagger}$} & \multicolumn{3}{|c|}{ Controls } & \multirow[t]{2}{*}{$P$-value } \\
\hline & II $(n=70)$ & IM (n=84) & MM $(n=49)$ & & II $(n=94)$ & IM $(n=90)$ & $M M(n=18)$ & \\
\hline Age (years) & $47.6 \pm 13.3$ & $46.9 \pm 13.3$ & $45.8 \pm 14.3$ & $>0.10$ & $40.2 \pm 12.8$ & $42.7 \pm 12.9$ & $46.6 \pm 14.7$ & 0.04 \\
\hline BMI $\left(\mathrm{kg} / \mathrm{m}^{2}\right)$ & $26.8 \pm 4.4$ & $27.1 \pm 5.3$ & $26.7 \pm 5.3$ & $>0.10$ & $23.0 \pm 3.1$ & $23.8 \pm 3.9$ & $24.2 \pm 2.9$ & $>0.10$ \\
\hline ALT (IU/L) & $32.2 \pm 22.5$ & $38.2 \pm 28.1$ & $72.2 \pm 59.0$ & $1.1 \times 10^{-7}$ & $17.4 \pm 9.2$ & $16.5 \pm 7.1$ & $17.5 \pm 5.6$ & $>0.10$ \\
\hline AST (IU/L) & $28 \pm 20.7$ & $30.7 \pm 23.8$ & $37.5 \pm 40.5$ & 0.07 & $19.9 \pm 4.8$ & $19.7 \pm 3.8$ & $19.3 \pm 3.6$ & $>0.10$ \\
\hline GGT (IU/L) & $73.1 \pm 52.1$ & $56.1 \pm 58.2$ & $52.8 \pm 46.6$ & $>0.10$ & $25.3 \pm 17.1$ & $24.5 \pm 16.0$ & $23.4 \pm 12.9$ & $>0.10$ \\
\hline Triglycerides (mmol/L) & $1.9 \pm 1.3$ & $2.2 \pm 1.5$ & $2.0 \pm 1.4$ & $>0.10$ & $1.0 \pm 0.5$ & $1.1 \pm 0.5$ & $1.2 \pm 0.4$ & $>0.10$ \\
\hline LDL-C (mmol/L) & $3.6 \pm 1.2$ & $3.4 \pm 0.9$ & $3.0 \pm 1.0$ & $4.1 \times 10^{-3}$ & $2.5 \pm 1.1$ & $2.6 \pm 1.1$ & $2.8 \pm 1.2$ & $>0.10$ \\
\hline $\mathrm{HDL}-\mathrm{C}(\mathrm{mmol} / \mathrm{L})$ & $1.3 \pm 0.5$ & $1.3 \pm 0.7$ & $1.4 \pm 0.6$ & $>0.10$ & $1.9 \pm 0.8$ & $1.9 \pm 1.0$ & $2.0 \pm 1.1$ & $>0.10$ \\
\hline FBG (mmol/L ) & $6.0 \pm 1.1$ & $6.1 \pm 1.5$ & $6.0 \pm 1.1$ & $>0.10$ & $5.5 \pm 1.3$ & $5.6 \pm 1.4$ & $5.9 \pm 0.9$ & $>0.10$ \\
\hline
\end{tabular}

${ }^{\dagger}$ Data are described by mean \pm standard deviation.

${ }^{\ddagger}$ Nominal $P$-values were calculated by fitting linear regression models, in which Age and BMI were adjusted for when testing other variables. After Bonferroni multiple testing correction (18 tests), the only significant association was between ALT levels and genotype in cases (adjusted $P$-value $=2.0 \times 10^{-6}$ ).

significant association between them $[15,16]$, despite that I148M was associated with fibrosis progression in both studies. In this study we reported a positive association result between I148M and the ultrasonographydetermined steatosis grade in an East Asian population.

One major limitation of this study is that the steatosis grade was assessed based on ultrasonography instead of liver biopsy, which represents the best diagnostic test for fatty liver diseases. In this study, the steatosis degree was graded by an experienced hepatologist according to established criteria [26], which showed an accuracy of
$88 \%$ in the diagnosis and staging of fatty liver from a direct comparison between the pathological and ultrasonographic findings [38]. Thus the association between I148M and the ultrasonography-determined steatosis degree was unlikely false positive due to technology limitations.

Plasma liver-enzyme levels are widely used as indicators of liver damage and they are influenced by environmental and genetic factors. ALT levels were shown to be associated with the PNPLA3 I148M variant $[11,39,40]$, and we replicated this association in cases but not in

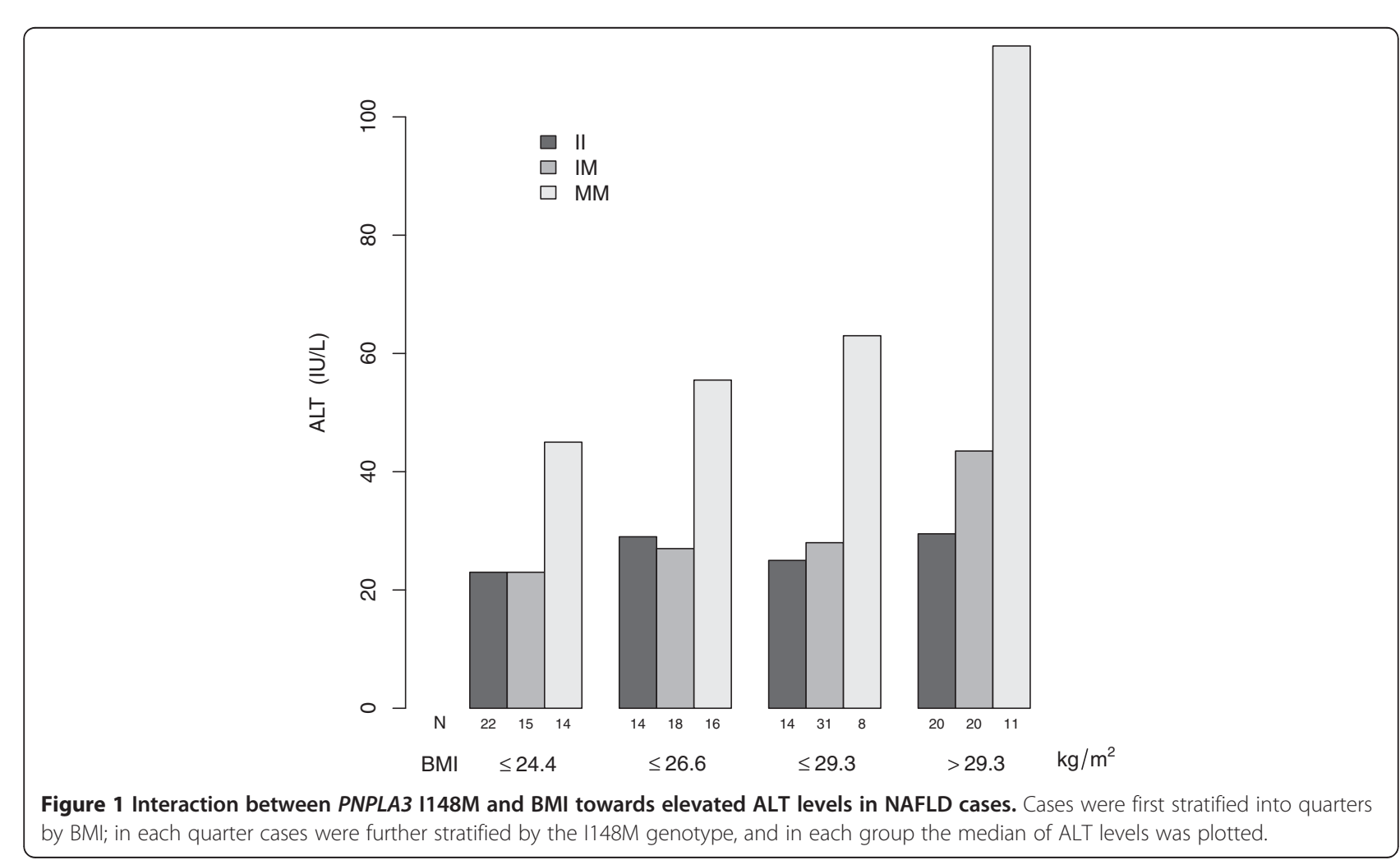


controls. ALT levels were more variable in cases than in controls (Table 2), which partially explained the difference of the association results. We did not merge cases and controls together for an association test because of heteroscedasticity.

Although the association of PNPLA3 with hepatic fat content and liver function is well established, its involvement in lipoprotein metabolism remains indeterminate. In this study the $148 \mathrm{M}$ allele was associated with reduced LDL-C levels in the NAFLD patients (nominal $P$-value $=4.1 \times 10^{-3}$ ); when the ALT levels were adjusted for, the association became more significant $(P$-value $=$ $3.6 \times 10^{-3}$ ). This result was consistent with that in another large study [41], implying the association between I148M and LDL-C levels was not simply a consequence of liver function impairment. In silico bioinformatic analysis suggested that PNPLA3 be involvoed in the metabolism of apoB-containing lipoproteins [41]. Although in vitro experiments suggested that PNPLA3 be involved in triglycerides metabolism [11,42], there was no association between $1148 \mathrm{M}$ and triglycerides either in normal populations [11] or in our NAFLD cases $(P$-value $>0.10)$. However, there were reports on their association in obese populations [25,41]. We speculate that I148M interacts with BMI / body fat distribution on triglyceride levels.

It is intriguing to investigate whether metabolic comorbidities such as obesity and insulin resistance, which are associated with the pathogenesis of NAFLD, are also influenced by the PNPLA3 I148M variant. There was no significant association between I148M and BMI in either cases or controls, consistent with the results in general populations $[11,13]$. It was suggested that body fat distribution, in particular abdominal fat, be implicated in the risk of developing NAFLD [43]. There were studies showing interaction between I148M and obesity parameters (BMI and waist circumference) on ALT levels [21,24] and type 2 diabetes susceptibility [25]. In this study we observed that the MM genotype interacted with high BMI to elevate ALT levels in cases; however, their interaction towards NAFLD susceptibility was insignificant by either a case-only or a case-control study. Larger sample studies are needed to investigate whether this interaction contributes to NAFLD susceptibility and triglyceride levels.

\section{Competing interests}

The authors declare no competing interest.

\section{Authors' contributions}

YL and ZT carried out clinical studies; CX and HK carried out data analysis; YL and CX were in charge of manuscript writing and project supervising. All authors read and approved the final manuscript.

\section{Acknowledgements}

We thank the reviewers for constructive comments that tremendously improved the manuscript. We are grateful to the participants of the study. YL is supported by the Liaoning Science and Technology Grant No. 2011225015, and $C X$ is supported by the American Heart Association Scientist Development Grant No. 10SDG4220051.

\section{Author details}

'Department of Gastroenterology, First Affiliated Hospital of China Medical University, Shenyang 110001, China. ${ }^{2}$ McDermott Center for Human Growth and Development, University of Texas Southwestern Medical Center at Dallas, Dallas, TX 75390, USA. ${ }^{3}$ Department of General Surgery, Shengjing Hospital of China Medical University, Shenyang 110001, China.

Received: 24 May 2012 Accepted: 24 October 2012

Published: 23 November 2012

\section{References}

1. Cohen JC, Horton JD, Hobbs HH: Human fatty liver disease: old questions and new insights. Science 2011, 332:1519-1523.

2. Omagari K, Kadokawa Y, Masuda J, Egawa I, Sawa T, Hazama H, Ohba K, Isomoto H, Mizuta Y, Hayashida K, et al: Fatty liver in non-alcoholic non-overweight Japanese adults: incidence and clinical characteristics. J Gastroenterol Hepatol 2002, 17:1098-1105.

3. Park SH, Jeon WK, Kim SH, Kim HJ, Park DI, Cho YK, Sung IK, Sohn Cl, Keum DK, Kim Bl: Prevalence and risk factors of non-alcoholic fatty liver disease among Korean adults. J Gastroenterol Hepatol 2006, 21:138-143.

4. Fan JG, Farrell GC: Epidemiology of non-alcoholic fatty liver disease in China. J Hepatol 2009, 50:204-210.

5. Chitturi S, Farrell GC, George J: Non-alcoholic steatohepatitis in the Asia-Pacific region: future shock? J Gastroenterol Hepatol 2004, 19:368-374.

6. Fan JG, Saibara T, Chitturi S, Kim BI, Sung JJ, Chutaputti A: What are the risk factors and settings for non-alcoholic fatty liver disease in Asia-Pacific? J Gastroenterol Hepatol 2007, 22:794-800.

7. Ludwig J, Viggiano TR, MCGill DB, Oh BJ: Nonalcoholic steatohepatitis: Mayo Clinic experiences with a hitherto unnamed disease. Mayo Clin Proc 1980, 55:434-438.

8. Struben VM, Hespenheide EE, Caldwell SH: Nonalcoholic steatohepatitis and cryptogenic cirrhosis within kindreds. Am J Med 2000, 108:9-13.

9. Schwimmer JB, Celedon MA, Lavine JE, Salem R, Campbell N, Schork NJ, Shiehmorteza M, Yokoo T, Chavez A, Middleton MS, et al Heritability of nonalcoholic fatty liver disease. Gastroenterology 2009, 136:1585-1592.

10. Hernaez R: Genetic factors associated with the presence and progression of nonalcoholic fatty liver disease: a narrative review. Gastroenterol Hepatol 2012, 35:32-41.

11. Romeo S, Kozlitina J, Xing C, Pertsemlidis A, Cox D, Pennacchio LA, Boerwinkle E, Cohen JC, Hobbs HH: Genetic variation in PNPLA3 confers susceptibility to nonalcoholic fatty liver disease. Nat Genet 2008, 40:1461-1465.

12. Chalasani N, Guo X, Loomba R, Goodarzi MO, Haritunians T, Kwon S, Cui J, Taylor KD, Wilson L, Cummings OW, et al: Genome-wide association study identifies variants associated with histologic features of nonalcoholic Fatty liver disease. Gastroenterology 2010, 139:1567-1576. 1576 e1561-1566.

13. Speliotes EK, Yerges-Armstrong LM, Wu J, Hernaez R, Kim LJ, Palmer CD, Gudnason V, Eiriksdottir G, Garcia ME, Launer L, et al: Genome-wide association analysis identifies variants associated with nonalcoholic fatty liver disease that have distinct effects on metabolic traits. PLoS Genet 2011, 7:e1001324.

14. Romeo S, Huang-Doran I, Baroni MG, Kotronen A: Unravelling the pathogenesis of fatty liver disease: patatin-like phospholipase domain-containing 3 protein. Curr Opin Lipidol 2010, 21:247-252.

15. Hotta K, Yoneda M, Hyogo H, Ochi H, Mizusawa S, Ueno T, Chayama K Nakajima A, Nakao K, Sekine A: Association of the rs738409 polymorphism in PNPLA3 with liver damage and the development of nonalcoholic fatty liver disease. BMC Med Genet 2010, 11:172.

16. Li X, Zhao Q, Wu K, Fan D: I148M variant of PNPLA3 confer increased risk for nonalcoholic fatty liver disease not only in European population, but also in Chinese population. Hepatology 2011, 54:2275.

17. Li Y, Xing C, Cohen JC, Hobbs HH: Genetic variant in PNPLA3 is associated with nonalcoholic fatty liver disease in China. Hepatology 2012, 55:327-328.

18. Wang CW, Lin HY, Shin SJ, Yu ML, Lin ZY, Dai CY, Huang JF, Chen SC, Li SS, Chuang WL: The PNPLA3 I148M polymorphism is associated with insulin resistance and nonalcoholic fatty liver disease in a normoglycaemic population. Liver Int 2011, 31:1326-1331. 
19. Kawaguchi T, Sumida Y, Umemura A, Matsuo K, Takahashi M, Takamura T, Yasui K, Saibara T, Hashimoto E, Kawanaka M, et al: Genetic Polymorphisms of the human PNPLA3 gene are strongly associated with severity of non-alcoholic fatty liver disease in japanese. PLoS One 2012, 7:e38322.

20. Sookoian S, Pirola CJ: Meta-analysis of the influence of I148M variant of patatin-like phospholipase domain containing 3 gene (PNPLA3) on the susceptibility and histological severity of nonalcoholic fatty liver disease. Hepatology 2011, 53:1883-1894.

21. Romeo S, Sentinelli F, Dash S, Yeo GS, Savage DB, Leonetti F, Capoccia D, Incani M, Maglio C, lacovino M, et al: Morbid obesity exposes the association between PNPLA3 I148M (rs738409) and indices of hepatic injury in individuals of European descent. Int J Obes (Lond) 2010, 34:190-194.

22. Romeo S, Sentinelli F, Cambuli VM, Incani M, Congiu T, Matta V, Pilia S, Huang-Doran I, Cossu E, Loche S, et al: The 148M allele of the PNPLA3 gene is associated with indices of liver damage early in life. $J$ Hepatol 2010, 53:335-338.

23. Lin YC, Chang PF, Hu FC, Yang WS, Chang MH, Ni YH: A common variant in the PNPLA3 gene is a risk factor for non-alcoholic fatty liver disease in obese Taiwanese children. J Pediatr 2011, 158:740-744.

24. Giudice EM, Grandone A, Cirillo G, Santoro N, Amato A, Brienza C, Savarese $P$, Marzuillo P, Perrone L: The association of PNPLA3 variants with liver enzymes in childhood obesity is driven by the interaction with abdominal fat. PLoS One 2011, 6:e27933.

25. Palmer CN, Maglio C, Pirazzi C, Burza MA, Adiels M, Burch L, Donnelly LA, Colhoun $\mathrm{H}$, Doney AS, Dillon JF, et al: Paradoxical lower serum triglyceride levels and higher type 2 diabetes Mellitus susceptibility in obese individuals with the PNPLA3 148M variant. PLoS One 2012, 7:e39362.

26. Zeng MD, Fan JG, Lu LG, Li YM, Chen CW, Wang BY, Mao YM: Guidelines for the diagnosis and treatment of nonalcoholic fatty liver diseases. J Dig Dis 2008, 9:108-112.

27. Luan JA, Wong MY, Day NE, Wareham NJ: Sample size determination for studies of gene-environment interaction. Int J Epidemiol 2001, 30:1035-1040.

28. Garcia-Closas M, Lubin JH: Power and sample size calculations in case-control studies of gene-environment interactions: comments on different approaches. Am J Epidemiol 1999, 149:689-692.

29. Lubin $\mathrm{JH}$, Gail MH: On power and sample size for studying features of the relative odds of disease. Am J Epidemiol 1990, 131:552-566.

30. VanderWeele TJ: Sample size and power calculations for case-only interaction studies. Epidemiology 2011, 22:873-874.

31. R Development Core Team: R: A language and environment for statistical computing. R Foundation for Statistical Computing. Vienna: Austria; 2011.

32. Wittke-Thompson JK, Pluzhnikov A, Cox NJ: Rational inferences about departures from Hardy-Weinberg equilibrium. Am J Hum Genet 2005, 76:967-986

33. Sookoian S, Castano GO, Burgueno AL, Gianotti TF, Rosselli MS, Pirola CJ: A nonsynonymous gene variant in the adiponutrin gene is associated with nonalcoholic fatty liver disease severity. J Lipid Res 2009, 50:2111-2116.

34. Rotman Y, Koh C, Zmuda JM, Kleiner DE, Liang TJ: The association of genetic variability in patatin-like phospholipase domain-containing protein 3 (PNPLA3) with histological severity of nonalcoholic fatty liver disease. Hepatology 2010, 52:894-903.

35. Speliotes EK, Butler JL, Palmer CD, Voight BF, Hirschhorn JN: PNPLA3 variants specifically confer increased risk for histologic nonalcoholic fatty liver disease but not metabolic disease. Hepatology 2010, 52:904-912.

36. Valenti L, Al-Serri A, Daly AK, Galmozzi E, Rametta R, Dongiovanni $P$, Nobili V, Mozzi E, Roviaro G, Vanni E, et al: Homozygosity for the patatin-like phospholipase-3/adiponutrin I148M polymorphism influences liver fibrosis in patients with nonalcoholic fatty liver disease. Hepatology 2010, 51:1209-1217.

37. Valenti L, Alisi A, Galmozzi E, Bartuli A, Del Menico B, Alterio A Dongiovanni P, Fargion S, Nobili V: I148M patatin-like phospholipase domain-containing 3 gene variant and severity of pediatric nonalcoholic fatty liver disease. Hepatology 2010, 52:1274-1280.

38. Needleman L, Kurtz AB, Rifkin MD, Cooper HS, Pasto ME, Goldberg BB: Sonography of diffuse benign liver disease: accuracy of pattern recognition and grading. Am J Roentgenol 1986, 146:1011-1015.

39. Yuan X, Waterworth D, Perry JR, Lim N, Song K, Chambers JC, Zhang W, Vollenweider $\mathrm{P}$, Stirnadel $\mathrm{H}$, Johnson $\mathrm{T}$, et al: Population-based genome-wide association studies reveal six loci influencing plasma levels of liver enzymes. Am J Hum Genet 2008, 83:520-528.

40. Chambers JC, Zhang W, Sehmi J, Li X, Wass MN, Van der Harst P, Holm H, Sanna S, Kavousi M, Baumeister SE, et al: Genome-wide association study identifies loci influencing concentrations of liver enzymes in plasma. Nat Genet 2011, 43:1131-1138.

41. Kollerits B, Coassin S, Beckmann ND, Teumer A, Kiechl S, Doring A, Kavousi M, Hunt SC, Lamina C, Paulweber B, et al: Genetic evidence for a role of adiponutrin in the metabolism of apolipoprotein B-containing lipoproteins. Hum Mol Genet 2009, 18:4669-4676.

42. Lake AC, Sun Y, Li JL, Kim JE, Johnson JW, Li D, Revett T, Shih HH, Liu W, Paulsen JE, et al: Expression, regulation, and triglyceride hydrolase activity of Adiponutrin family members. J Lipid Res 2005, 46:2477-2487.

43. Manco M, Bedogni G, Marcellini M, Devito R, Ciampalini P, Sartorelli MR, Comparcola D, Piemonte F, Nobili V: Waist circumference correlates with liver fibrosis in children with non-alcoholic steatohepatitis. Gut 2008, 57:1283-1287.

doi:10.1186/1471-2350-13-113

Cite this article as: Li et al.: Genetic variant I148M in PNPLA3 is associated with the ultrasonography-determined steatosis degree in a Chinese population. BMC Medical Genetics 2012 13:113.

\section{Submit your next manuscript to BioMed Central and take full advantage of:}

- Convenient online submission

- Thorough peer review

- No space constraints or color figure charges

- Immediate publication on acceptance

- Inclusion in PubMed, CAS, Scopus and Google Scholar

- Research which is freely available for redistribution 